Tecno Lógicas

ISSN 0123-7799

Vol. 19, No. 37, pp. 29-43

Julio-diciembre de 2016

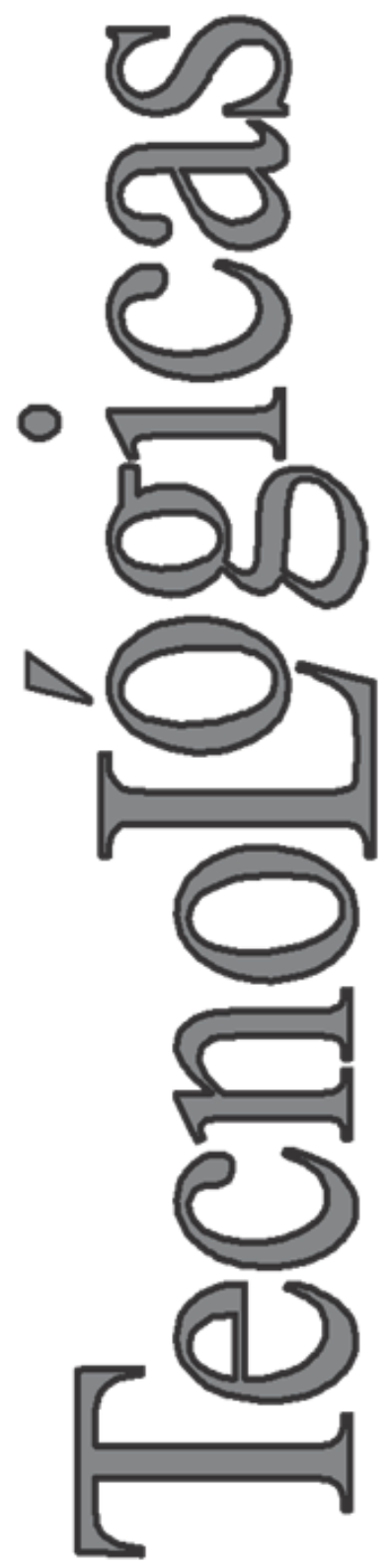

(C) Copyright 2015 por Autores y Tecno Lógicas Este trabajo está licenciado bajo una Licencia Internacional Creative Commons Atribución (CC BY)

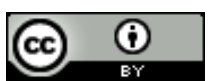

\section{Análisis espectral a través de bancos de filtros aplicado al pre-procesamiento para la umbralización de señales de pulso oximetría}

\section{Spectral analysis through filter banks aplied to preprocessing oriented to thresholding of pulse oximetry signal}

\author{
Javier E. González-Barajas ${ }^{1}$, \\ Cristian C. Velandia ${ }^{2}$, Jeysson Lyma-Guaqueta ${ }^{3}$ y \\ Pedro Ospina-Fuentes ${ }^{4}$
}

Recibido: 16 de noviembre de 2015 ,

Aceptado: 12 de mayo de 2016

Cómo citar / How to cite

J.E. González-Barajas, C.C. Velandia, J. Lyma-Guaqueta y P. OspinaFuentes, "Análisis espectral a través de bancos de filtros aplicado al pre-procesamiento para la umbralización de señales de pulso oximetría”, Tecno Lógicas, vol. 19, no. 37, pp.29-43, 2016.

1 M. Sc. en Ingeniería UIS, Facultad de Ingeniería Electrónica, División de Ingenierías, Universidad Santo Tomás, BogotáColombia, javiergonzalezb@usantotomas.edu.co

2 Ing. Electrónico, USTA, Facultad de Ingeniería Electrónica, División de Ingenierías, Universidad Santo Tomás, Bogotá-Colombia, cristianvelandia@usantotomas.edu.co

3 Ing. Electrónico, USTA, Facultad de Ingeniería Electrónica, División de Ingenierías, Universidad Santo Tomás, Bogotá-Colombia, Jeyssonlyma@usantotomas.edu.co

4 Ing. Electrónico, USTA, Facultad de Ingeniería Electrónica, División de Ingenierías, Universidad Santo Tomás, Bogotá-Colombia, pedroospina@usantotomas.edu.co 


\title{
Resumen
}

La señal de pulso oximetría (SPO2) permite el cálculo del porcentaje de oxígeno en sangre y es adquirida en uno de los dedos del paciente. En condiciones normales, las variaciones de la señal SPO2 están correlacionadas con el ritmo cardiaco del paciente y su valor máximo está en fase con la onda R de la señal electrocardiográfica (ECG). Esta propiedad permite a la señal SPO2 ser la base para la estimación de la frecuencia cardiaca instantánea. Con la finalidad de poder medir la frecuencia cardiaca instantánea, a partir de la señal SPO2, es necesario un proceso de umbralización para la detección de los valores máximos, en fase con la onda $\mathrm{R}$ del complejo cardiaco. En este artículo se presenta un método iterativo para establecer la selección de frecuencias de corte para el diseño de filtros digitales, que permitan la detección de los valores máximos de la señal de pulso oximetría. Se presentan los resultados obtenidos a partir de la implementación de bancos de filtros y se demuestra su capacidad para obtener versiones de la señal de pulso oximetría y los valores de frecuencia de las componentes espectrales asociadas a los valores máximos de las señales de pulso oximetría. Los experimentos elaborados utilizaron señales de la base de datos CAPNOBASE que contienen señales SPO2 y ECG adquiridas simultáneamente. Los datos permitieron comprobar que los bancos de filtros permiten seleccionar la versión adecuada de señal SPO2 con picos positivos en fase con la onda $\mathrm{R}$ de la señal ECG.

\section{Palabras clave}

Pulso oximetría, banco de filtros, análisis espectral, frecuencia cardiaca.

\begin{abstract}
The pulse oximetry signal (SPO2), allows the calculation of the oxygen saturation level in the blood, and it is acquired over the index finger of the patient. Under normal conditions, the variations in SPO2 have correlated with heart rhythm and its maximum value is in phase with the $R$ wave in electrocardiographic signal (ECG). This property enables the SPO2 signal to be the basis for an alternative method for estimating the instantaneous heart rate. For measuring the instantaneous heart rate from the SPO2, it is necessary to carry out a signal thresholding process for detecting peak values in phase with the $R$-wave of the cardiac complex. In this paper, an iterative solution method is proposed to establish the cutoff frequency selection for the design of digital filters that allow detection of the maximum values of the signal pulse oximetry. The results obtained from the implementation of filter banks, demonstrated their ability to obtain versions of the pulse oximetry signal and frequency values of the spectral components, associated with the maximum values of the SPO2. Experiments used the CAPNOBASE processed signals database, which contains SPO2 and ECG signals, acquired simultaneously. The results allowed to verify that the filter bank allows to select the appropriate version of SPO2 signal with positive peaks, in phase with the $\mathrm{R}$ wave of the ECG signal.
\end{abstract}

\section{Keywords}

Pulse oximetry, filter bank, spectral analysis, heart rate. 


\section{INTRODUCCIÓN}

La señal de pulso oximetría permite estimar el porcentaje de oxígeno en la sangre (SPO2) y es adquirida en la superficie de uno de los dedos del paciente (comúnmente, el dedo índice). Para generar este registro se debe contar con una fuente de luz con longitud de onda cercano al infra rojo y puede provenir de un diodo emisor de luz (LED) [1]. Este método óptico es también utilizado para estimar la frecuencia cardiaca y este tipo de medición se denomina fotopletismografía (PPG) [2].

En el caso de la adquisición de la señal PPG por transmisión, la fuente de luz está posicionada en la parte posterior a la yema del dedo y el sensor en la parte contraria. También se cuenta con un tipo de adquisición por reflexión, en el cual la fuente de luz es de color verde y está en el mismo plano del sensor (yema del dedo) [3]. La Fig. 1 ilustra un ejemplo de un sistema de adquisición tradicional de la señal de pulso oximetría por transmisión.

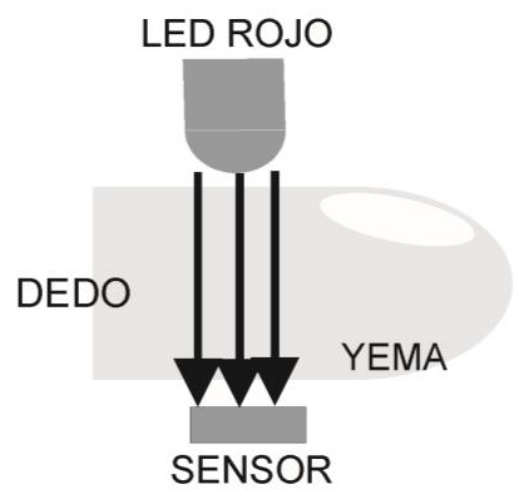

\section{TRANSMISIÓN}

Fig. 1. Adquisición de la señal de pulso oximetría: transmisión y reflexión. Fuente: autores.

Sin importar el método de adquisición de la señal de pulso oximetría, el rayo de luz adquirido por el sensor tendrá cambios de intensidad. Los niveles de intensidad de luz son proporcionales al porcentaje de oxigeno contenido en la sangre que circula a través de los vasos que componen el dedo índice [4]. La señal adquirida por el sensor permite obtener una curva de forma característica como la presentada en la Fig. 2.

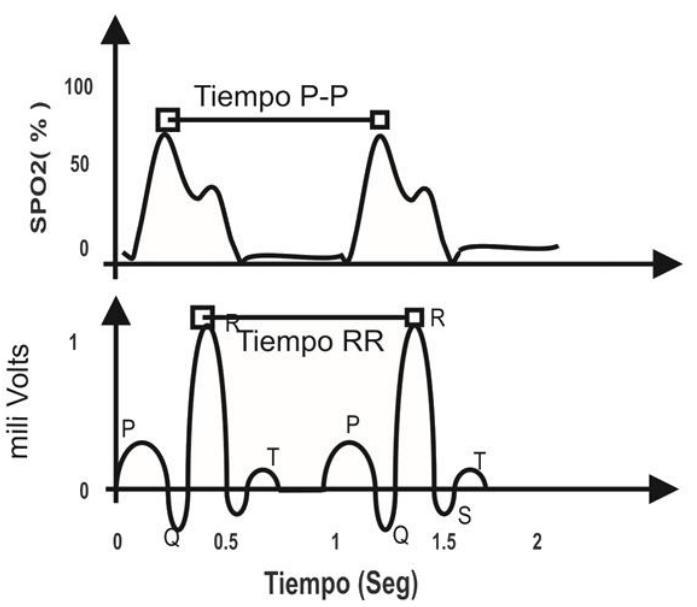

Fig. 2. Señal de pulso oximetría y el electrocardiograma. Fuente: autores.

De acuerdo con la Fig.2, la señal variable SPO2 presenta una curva característica con valores de picos positivos con un periodo determinado por el Tiempo P-P. Si la variable SPO2 es adquirida de manera simultánea con la señal electrocardiográfica (ECG), es posible comparar el intervalo de tiempo P-P con el Tiempo R-R, el cual corresponde al inverso matemático de la frecuencia cardiaca instantánea [5].

Trabajos anteriores han verificado la similitud entre el periodo de la curva SPO2 (Tiempo P-P) y el de la señal electrocardiográfica (Tiempo RR) [6]. Con base en esta similitud se considera que la curva SPO2 es una herramienta para la estimación de la frecuencia cardiaca instantánea [7]. La curva SPO2, contenida en la señal PPG, se ha consolidado como un insumo para $\mathrm{de}^{-}$ terminar variables cardiovasculares [8] y estimar el comportamiento de la señal electrocardiográfica [9]. También se ha convertido en una herramienta para estimar otras variables fisiológicas como el ritmo respiratorio [10], [11], oscilaciones gástricas [12], eventos en resucitación cardiopulmonar [13] y variaciones en condiciones de ejercicio [14]. 
La curva SPO2 requiere de diversas estrategias computaciones para ser extraída de la señal PPG, como es el caso del análisis espectral [15]. En el proceso de extracción de información pueden aparecer distorsiones causadas por artefactos [16] que pueden ser tratadas con técnicas como los filtros de partículas [17].

Desde el punto de vista de las técnicas de umbralización de la señal SPO2, la literatura reporta aplicaciones basadas en el cálculo del área bajo la curva del pico positivo [18]. También, umbralizaciones comparadas con el registro ECG [19] y el uso de filtros derivadores [20]. De acuerdo con esta revisión, el procesamiento previo a la umbralización puede resumirse en la Fig. 3.

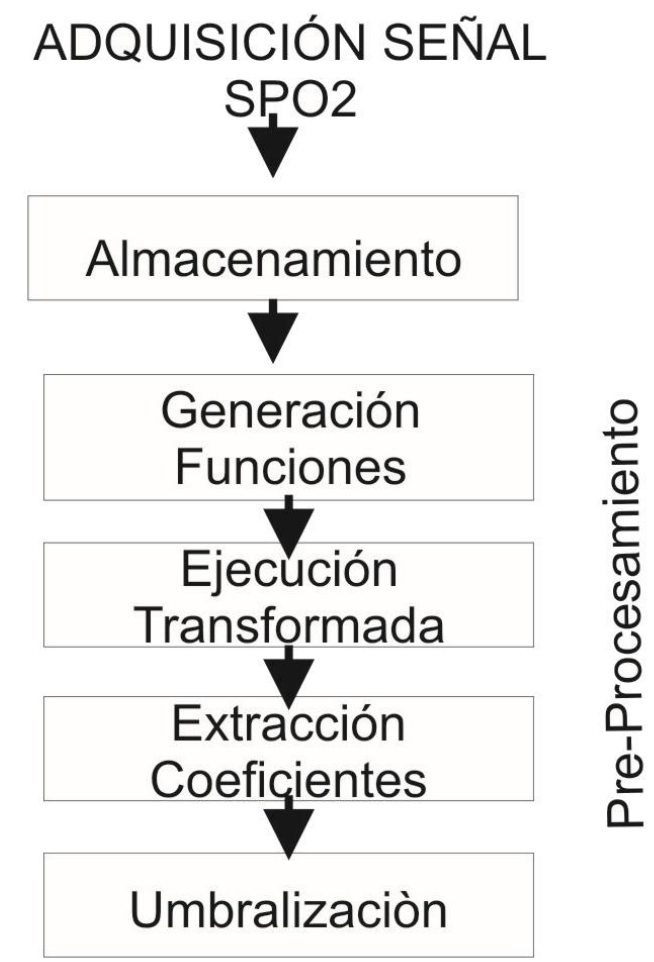

Fig. 3. Proceso de umbralización de la señal SPO2. Fuente: autores.

Como se puede apreciar en la Fig. 3, es indispensable acudir a transformadas de tiempo y frecuencia o segmentación en el dominio de la frecuencia, como pasos previos a la umbralización de la señal SPO2.
El proceso de umbralización de la señal SPO2 requiere del conocimiento de la información contenida en su espectro. El ancho de banda de la señal SPO2 está contenido entre las frecuencias 0 y $12 \mathrm{~Hz}$. Donde la frecuencia de oscilación se puede localizar entre la frecuencia de 0 y $1.2 \mathrm{~Hz}$ [21].

La umbralización de la señal SPO2 da la oportunidad de obtener una medida de tiempo entre sus picos positivos (Tiempo PP) y ser el insumo para la estimación de la frecuencia cardiaca instantánea [22], [23]. Esta medida es de gran importancia en dispositivos electrónicos para el monitoreo de pacientes [24].

El propósito de este artículo es diseñar una metodología de pre-procesamiento de señales SPO2 para las mejoras del proceso de umbralización. El bloque de preprocesamiento permite aislar las componentes espectrales de la señal SPO2 que no aportan información en la detección de picos positivos. Este bloque se ha diseñado través de bancos de filtros en el dominio del tiempo, que cumplen la tarea de proporcionar versiones modificadas de la señal SPO2. En este trabajo se resalta la ventaja que ofrece el banco de filtros, en cuanto a ser una herramienta iterativa que permite obtener diferentes versiones, desde una señal de entrada. Cada versión permite asociar características de la señal presentes en el dominio del tiempo.

Este artículo da a conocer el diseño de la estrategia de bancos de filtros y lo experimentos realizados para corroborar su funcionamiento. Están expuestos los experimentos que aportaron resultados para la selección del orden de los filtros digitales, y la relación entre frecuencias de corte del banco de filtros y las características de la señal SPO2. Por último, son presentados los resultados obtenidos en la detección de picos positivos de la señal SPO2, con comparaciones de detecciones de la onda $\mathrm{R}$ de la señal electrocardiográfica. 


\section{METOdOLOGíA}

Los experimentos llevados a cabo en este trabajo fueron realizados usando señales de pulso oximetría y electrocardiografía adquiridas simultáneamente. Las señales están almacenadas en la base de datos de uso libre CAPNOBASE [25]. Esta base de datos ha sido el principal insumo en diferentes trabajos orientados a la estimación de parámetros basados en SPO2 y PPG [26]. Las señales adquiridas poseen una frecuencia de muestreo $\mathrm{Fs}_{\mathrm{s}}=300 \mathrm{~Hz}$, longitud promedio de datos $\mathrm{N}=144.000$ mues $^{-}$ tras. La base de datos cuenta con 46 archivos almacenados en formato. mat, los cuales pueden ser manejados desde el asistente matemático Matlab.

La Fig. 4 ilustra un ejemplo de uno de los registros de CAPNOBASE, tomado de prueba. En este ejemplo se pueden observar 2000 muestras de dos señales adquiridas simultáneamente del mismo paciente: SPO2 y ECG.

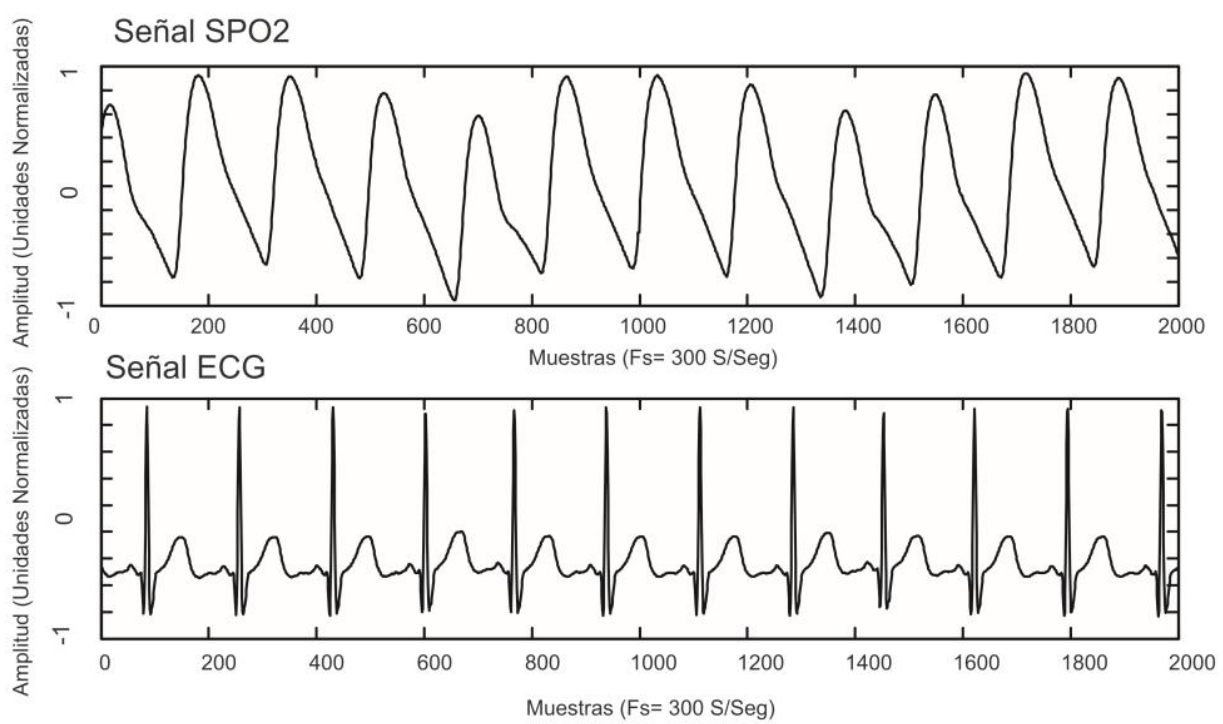

Fig. 4. Señal SPO2 y ECG adquiridas simultáneamente. Fuente: autores.

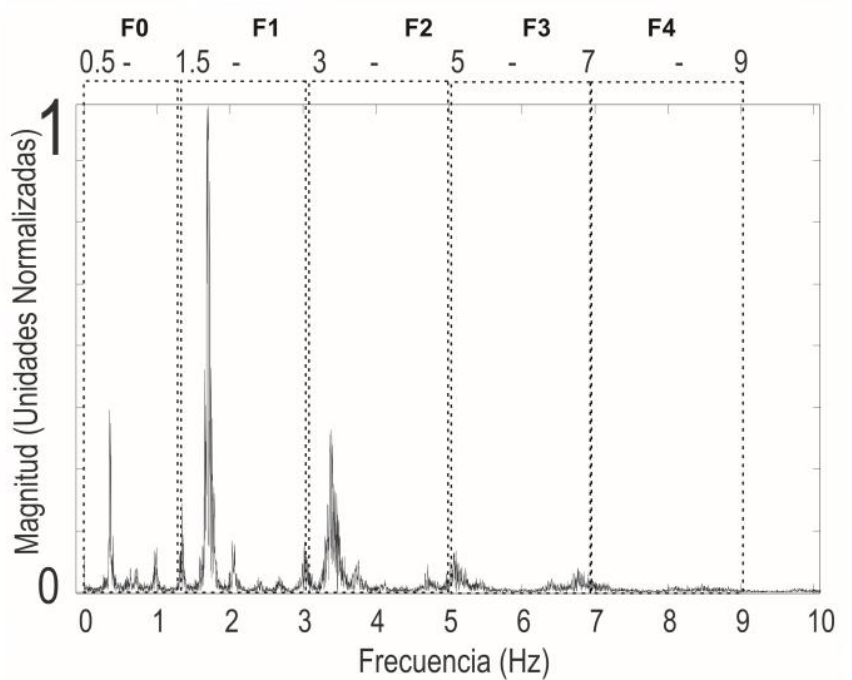

Fig. 5. Magnitud del espectro en potencia de la señal SPO2. Fuente: autores. 
La Fig. 5 contiene la magnitud del espectro en potencia de la señal SPO2 (unidades normalizadas). Se puede observar que el espectro está dividido en cinco rangos de frecuencia. En la Fig. 4 se puede apreciar que la magnitud del espectro en potencia de la señal SPO2 posee picos característicos, por lo cual se ha dividido en cinco regiones de interés delimitados por valores de frecuencia de corte.

Trabajos previos han permitido relacionar empíricamente cada banda expuesta en la Fig. 5, con características de la señal SPO2 [21].

\subsection{Diseño del banco de filtros}

El banco de filtros es una estrategia computacional que está compuesta por algoritmos de filtrado pasa-banda con frecuencias de cortes consecutivas. El propósito de un sistema basado en banco de filtros (Fig. 6) es tomar como señal de entrada un registro de SPO2 y obtener un grupo de señales de salida. Cada una de las salidas es una versión de la señal de entrada con un ancho de banda restringido. Las señales de salida poseen características propias, en el dominio del tiempo, asociadas a las componentes espectrales contenidas en el respectivo ancho de banda del filtro que las origina. En este trabajo se pretende encontrar, iterativamente el grupo de componentes espectrales asociados a los picos positivos de la señal SPO2.

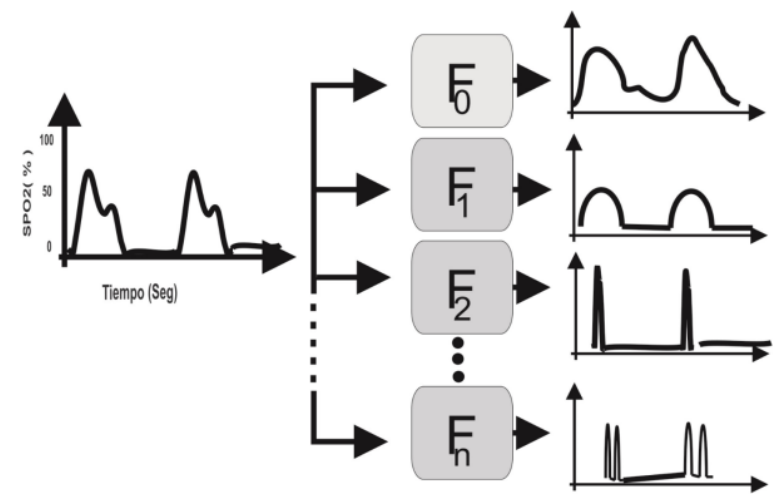

Fig. 6. Diagrama de bloques del banco de filtros. Fuente: autores.

Teniendo en cuenta artículos previamente publicados sobre el espectro de la señal SPO2 [21] y las regiones del espectro expuestas en Fig. 5, se establecen las frecuencias de corte de los diferentes filtros descritas en la Tabla 1.

\subsection{Implementación de algoritmos}

Los algoritmos que permitieron la ejecución de los experimentos han sido implementados bajo el asistente matemático Matlab. Para el diseño de los filtros se ha utilizado la técnica de la ventana [27], que permite calcular los coeficientes del filtro a través de la Eq. (1).

$B(k)=\left(\frac{\sin \left(2 \pi * f h * T s\left(k-\frac{L}{2}\right)\right)}{\left(\pi *\left(k-\frac{L}{2}\right)\right)}\right)-\left(\frac{\sin \left(2 \pi * f l * T s\left(k-\frac{L}{2}\right)\right)}{\left(\pi *\left(k-\frac{L}{2}\right)\right)}\right)$ 

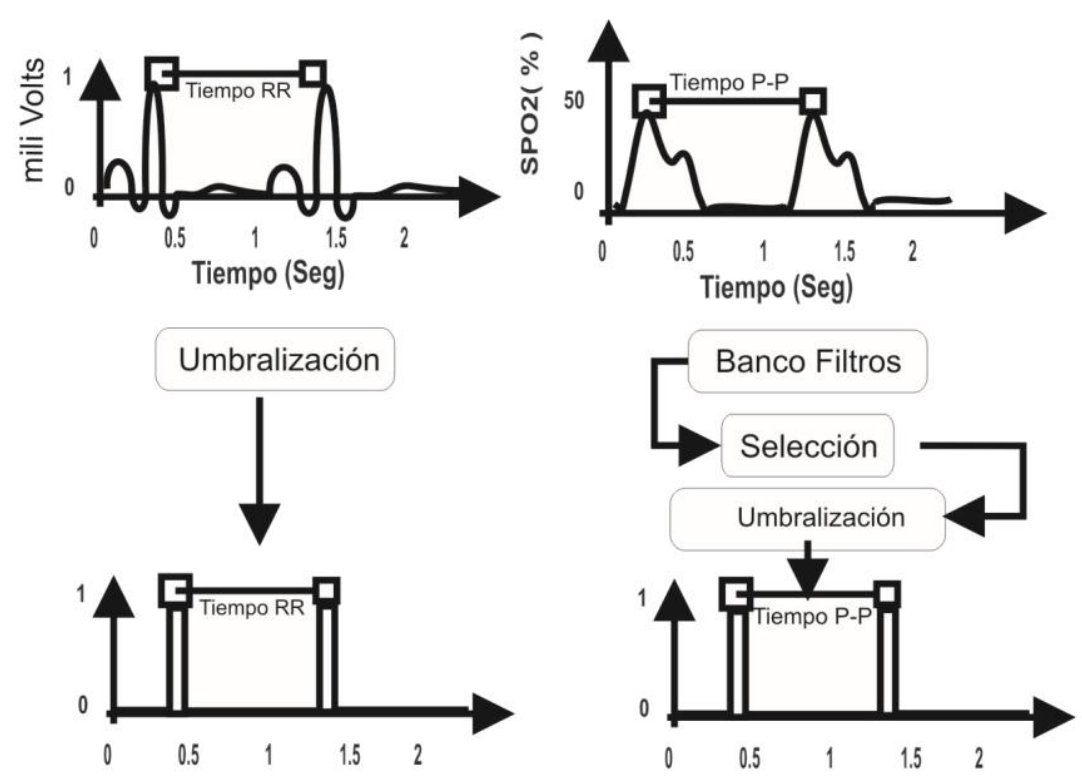

Fig. 7. Extracción de datos para generación de comparaciones. Fuente: autores.

donde los valores fh y fl, que corresponden a los valores de frecuencia de corte de alta y baja frecuencia, fueron tomados de la Tabla 1. El criterio para escoger la técnica de la ventana está basado en su practicidad de implementación y baja complejidad para el cálculo de los coeficientes.

El algoritmo implementado para la ejecución del banco de filtros carga el registro con señal SPO2 en formato .mat y queda almacenado en la variable X. Seguidamente se debe declarar el arreglo de datos que contiene los valores de frecuencias de corte de los filtros pasa banda: Fl(i) y $\mathrm{Fh}(\mathrm{i})$. De manera consecutiva son calculados los coeficientes del filtro pasa banda y almacenados en el arreglo B.

El algoritmo para la ejecución del bando de filtros está basado en la Ec. (2), que corresponde a la expresión de un filtro tipo FIR basado en ecuaciones en diferencias.

$$
Y(n)=\sum_{K=0}^{L-1} B(k) x(n-k)
$$

El orden del filtro L, puede ser determinado a través de experimentos iterativos en los cuales se compara el realce de la señal con el incremento de L.

En su última etapa, permite la visualización de las señales de salida de cada filtro pasa banda. El resultado final está almacenado en la matriz $\mathrm{Y}$, con una fila por cada señal de salida.

\subsection{Validación del experimento}

La validación del experimento está basada en la comparación de datos obtenidos a partir de la detección de ondas $\mathrm{R}$ (Señal ECG) y picos positivos de la señal SPO2. De cada proceso de detección se obtienen dos tablas de valores de tiempos: tiempos R- R y tiempos P-P (Ver Fig. 7).

La cantidad de señales SPO2 y ECG fue de 15 registros escogidos al azar y con una cantidad de 2000 muestras por cada una.

Las señales obtenidas de CAPNOBASE permiten obtener las señales SPO2 y ECG de un mismo paciente adquiridas de manera simultánea. Por tal razón, se obtiene como marco de referencia las ondas $\mathrm{R}$ de cada complejo cardiaco. Un bloque importante contenido en la Fig. 7 es el proceso de umbralización. Este método está basado en 
la técnica de umbralización a partir de operaciones sobre el histograma del ECG y permite obtener una onda cuadrada de valor binario ( 1 = detección de onda $\mathrm{R})$ [5]. El método de umbralización también es utilizado para la detección de los picos positivos de la señal SPO2.

La Fig 8. resume el proceso de umbralización utilizado para la detección de las ondas R y picos positivos (Señal SPO2). Este proceso inicia con la señal de entrada que es sometida a un filtrado pasa banda. En el caso de la señal ECG las frecuencias de corte del filtro pasa banda son obtenidos de trabajos previos [5] y son $\mathrm{Fl}=10 \mathrm{~Hz}$ y $\mathrm{Fh}=20 \mathrm{~Hz}$. El valor de filtrado pasa banda de la señal SPO2 es escogido a partir de los resultados obtenidos del banco de filtros. Después del filtrado pasa banda, se calcula el histograma de cada señal y su respectiva segunda derivación, a partir de la cual es estimado el valor umbral.

El proceso de umbralización ofrece como resultado dos arreglos de datos: Tiempos R- R y Tiempos P-P. A través de la Eq. (3) se calcula el error obtenido de cada medición de Tiempos P-P, tomando como referencia los Tiempos $\mathrm{R}-\mathrm{R}$, para el $\mathrm{k}^{-}$exi- $^{-}$ mo dato.

$$
\% E(k)=\frac{\mid \text { Tiempo }_{R-R}(k)-\text { Tiempo }_{p-p}(k) \mid}{\text { Tiempo }_{R-R}(k)} * 100 \%
$$

Para corroborar la importancia del uso del filtrado pasa banda se ha diseñado una prueba basada en la comparación de resultados positivos obtenidos de la señal SPO2 con y sin filtrado.

\section{RESULTADOS Y DISCUSIÓN}

Con la finalidad de poder encontrar el rango de frecuencias asociadas con la señal SPO2 del complejo cardiaco, se procede al análisis de esta señal bajo el esquema del banco de filtros. El resultado obtenido se puede observar en la Fig. 9.

Como se pudo observar en la Fig. 9, el filtro F2 permite obtener una versión de la señal SPO2 con un realce del pico máximo positivo. El filtro $\mathrm{F} 2$ posee una configuración pasa banda con $\mathrm{Fl}=3 \mathrm{~Hz}$ y $\mathrm{Fh}=5 \mathrm{~Hz}$. Este experimento permite establecer que el ancho de banda del filtro F2 reúne las componentes espectrales asociadas al pico positivo de mayor valor de la señal SPO2. Las señales obtenidas de los filtros F0, F1, F3 y F4, no presentan una señal SPO2 con un pico positivo que ofrezca la facilidad de un proceso de umbralización.

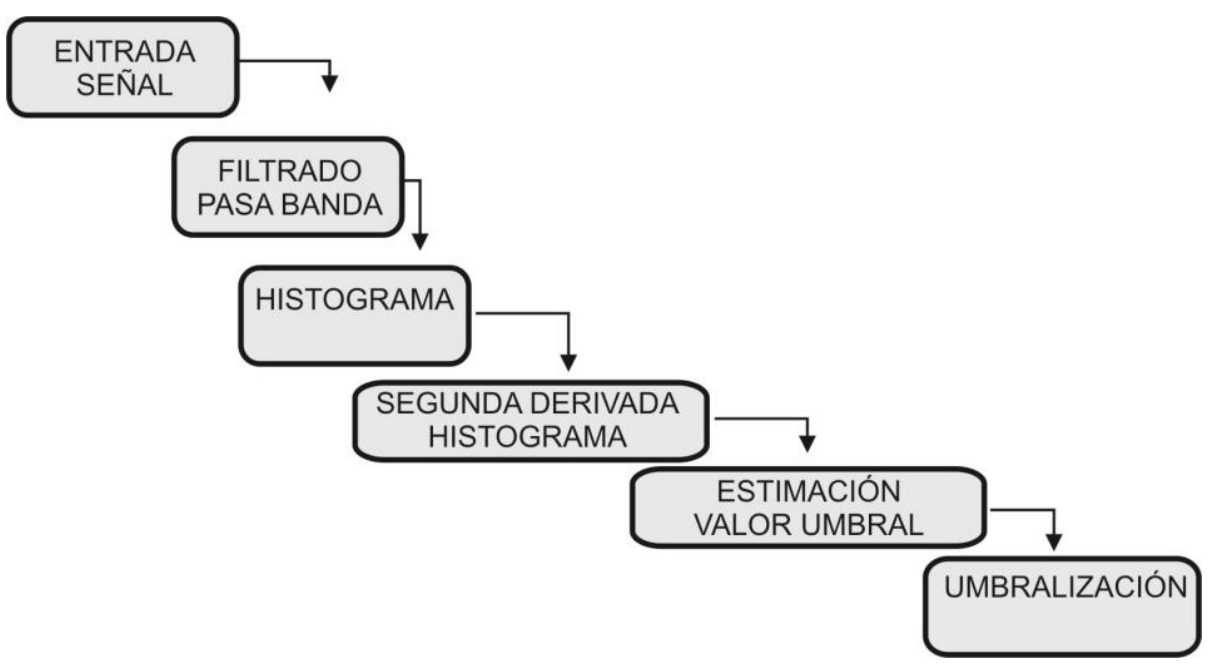

Fig. 8. Umbralización señales ECG y PPG.Fuente: autores. 


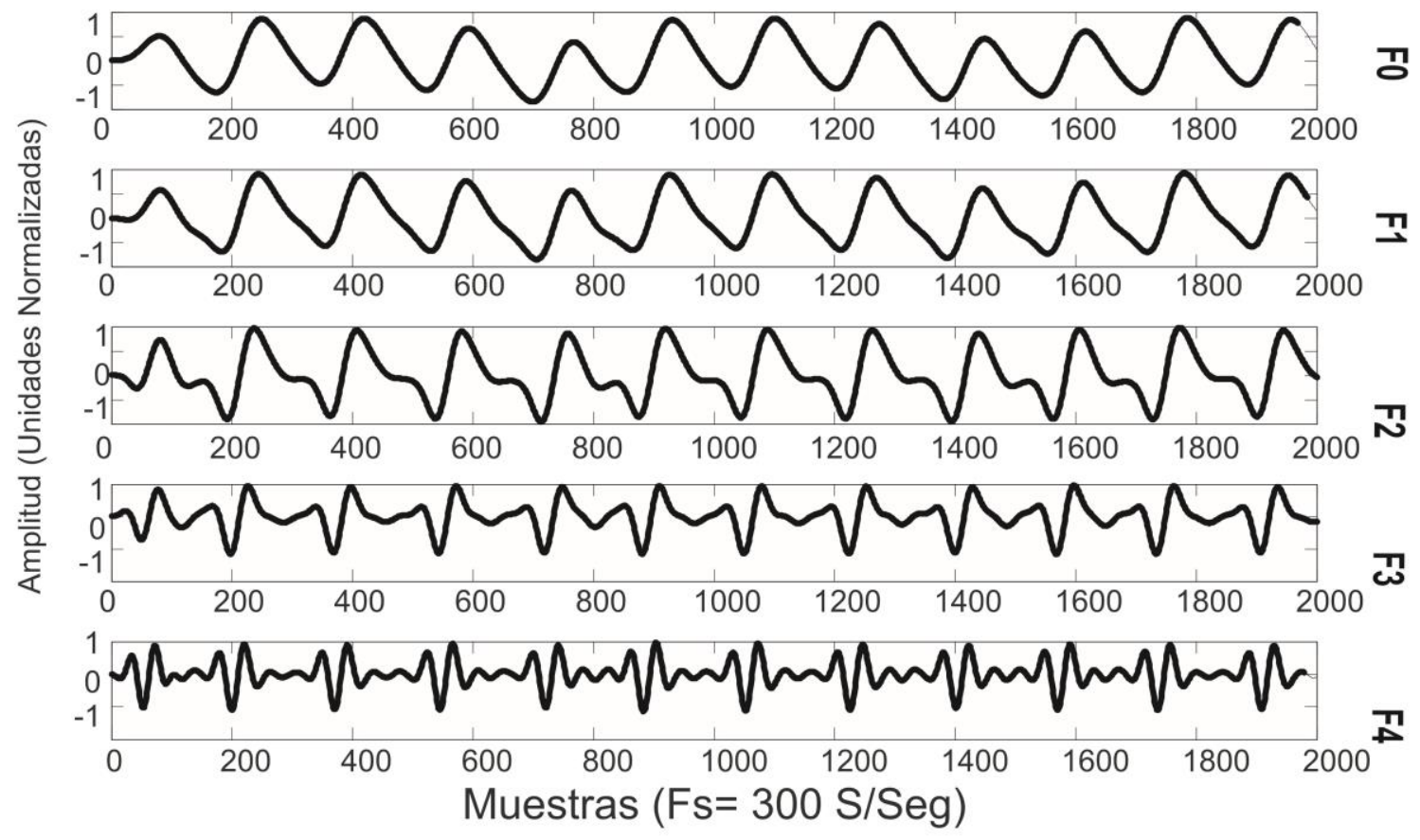

Fig. 9. Resultado de la ejecución del banco de filtros.

Fuente: autores.

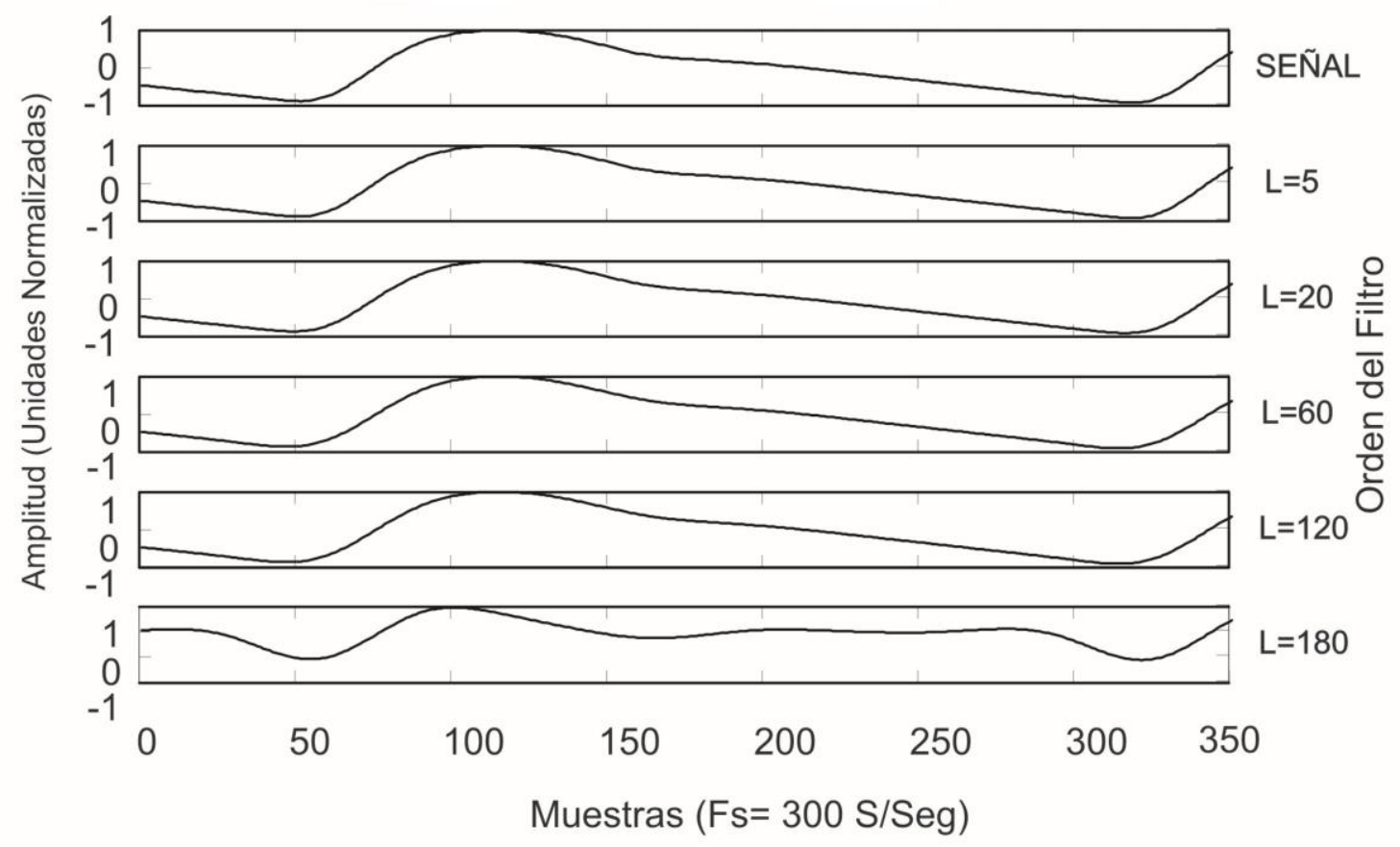

Fig. 10. Prueba de orden del Filtro. Fuente: autores. 

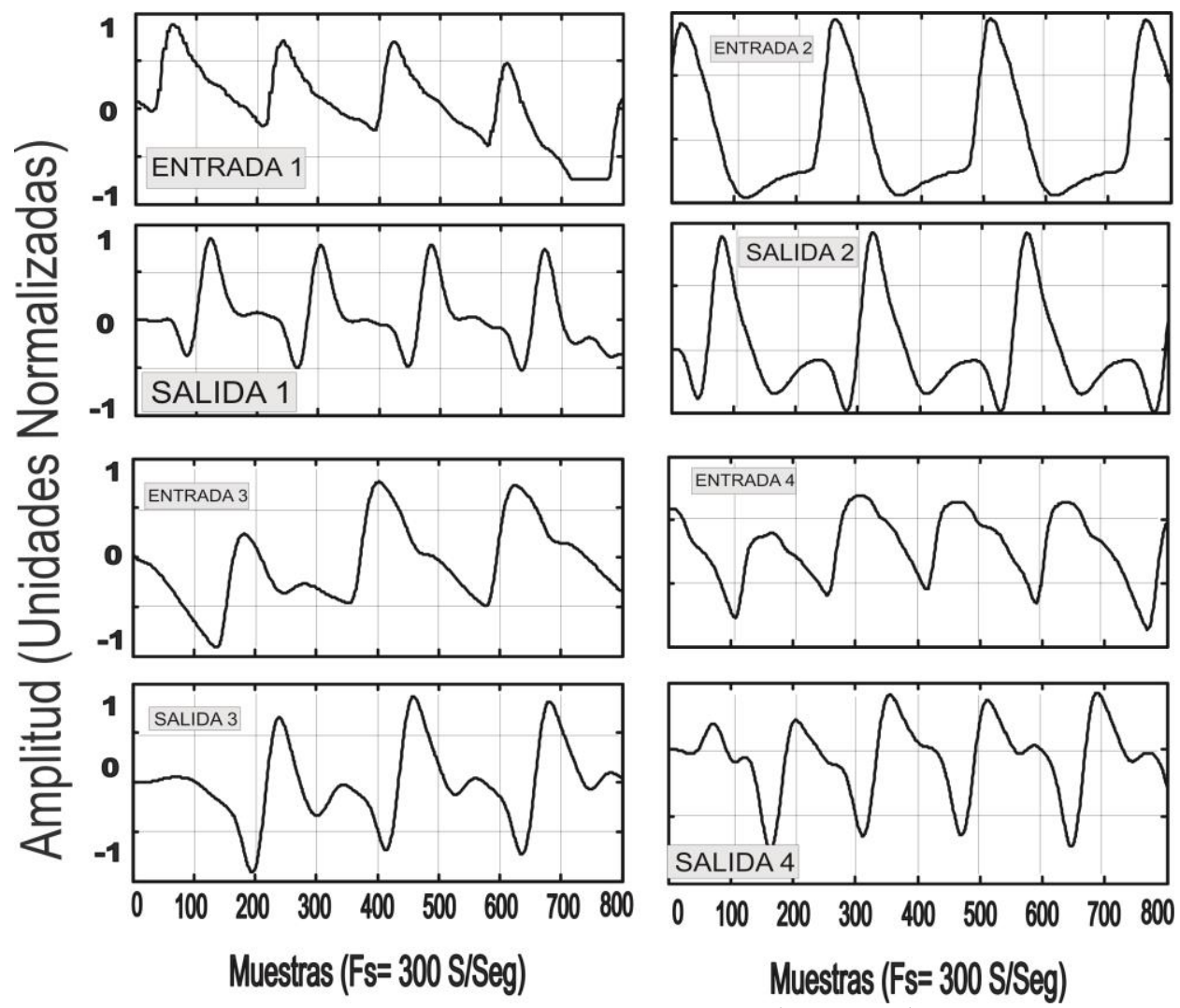

Fig. 11. Prueba realizada a cuatro señales $\mathrm{SPO} 2$ con el filtro F2 $(3 \mathrm{~Hz}-5 \mathrm{~Hz})$. Fuente: autores.

Para establecer el orden del filtro pasa banda, se realizó un experimento iterativo, el cual tuvo la finalidad de realizar pruebas con el filtrado pasa banda con diferentes valores de orden L. La Fig 10. Muestra los resultados obtenidos, como se puede observar que a partir del valor $\mathrm{L}=120$ ya se puede contar con un realce adecuado del pico positivo de la señal SPO2.

Para corroborar los resultados obtenidos a través del banco de filtros, se ha procedido a filtrar el resto de señales SPO2 con el filtro F2. El resultado obtenido se muestra en la Fig. 11, que contiene la salida del filtro obtenida con cuatro señales SPO2 de la base de datos CAPNOBASE. En cada uno de los ejemplos se puede ver la señal de entrada y su correspondiente señal de salida obtenida después de ejecutar el filtro F2.

Acorde a la metodología de validación, se ha realizado la umbralización a través del método basado en el histograma de las señales SPO2 obtenidas con el filtro F2 y de las señales ECG. En primera instancia se han cuantificado los aciertos de ondas detectadas, como por ejemplo el caso $\mathrm{ex}^{-}$ puesto en la Fig. 12. A continuación, se dará una breve descripción de la formulación del modelo que conduce al cálculo de las fuerzas que actúan sobre una partícula debido a su interacción con el fluido.

La Tabla 2 contiene el porcentaje de aciertos obtenidos. Se puede observar los ensayos realizados para cada uno de los 16 registros escogidos de la base de datos digital CAPNOBASE. Para cada ensayo fue tomada una ventana de 2000 muestras.

Tomando la Ec. (3), se han calculado los porcentajes de error de cada señal tomada de prueba. Los datos obtenidos están contenidos en la Tabla 3. La información que provee permite evaluar el grado de exactitud que ofrece el cálculo de intervalos de tiempo a través de la umbralización de la señal SPO2 tomando como referencia las ondas R de la señal ECG. 
Análisis espectral a través de bancos de filtros aplicado al pre-procesamiento para la umbralización de señales de pulso oximetría
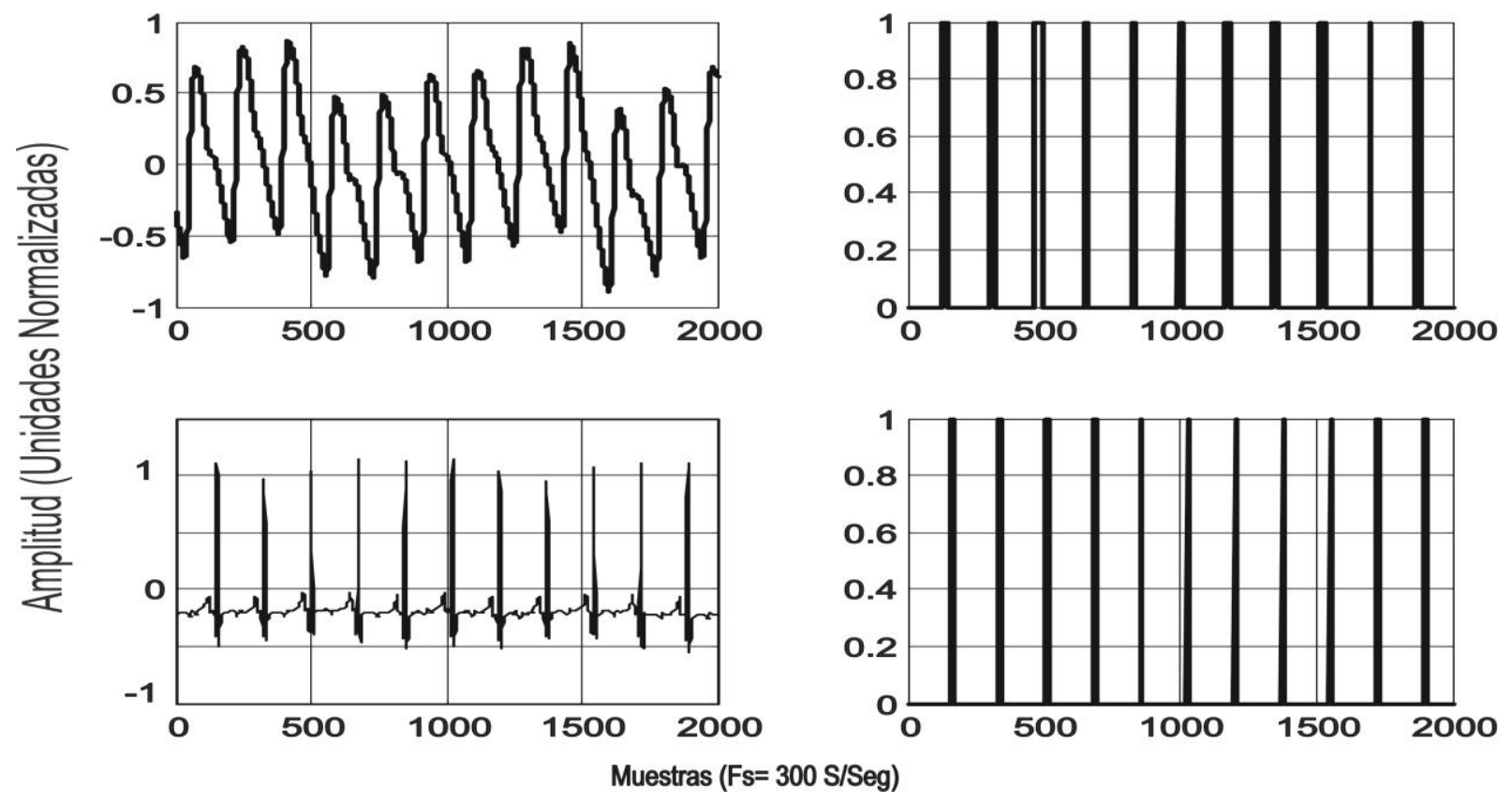

Fig. 12. Comparación de Eventos detectados. Fuente: autores.

Tabla 2. Porcentaje de aciertos. Fuente: autor.

\begin{tabular}{cccc}
\multicolumn{5}{c}{ Tabla 2. Porcentaje de aciertos. Fuente: autor. } \\
\hline Registro & $\begin{array}{c}\text { Ondas R } \\
\text { ECG }\end{array}$ & $\begin{array}{c}\text { Picos Positi } \\
\text { vos SPO2 }\end{array}$ & $\begin{array}{c}\text { Porcentaje } \\
\text { de aciertos }\end{array}$ \\
\hline 1 & 11 & 9 & 81,8 \\
2 & 13 & 13 & 100 \\
3 & 12 & 12 & 100 \\
4 & 11 & 11 & 100 \\
5 & 8 & 8 & 75 \\
6 & 8 & 6 & 75 \\
7 & 15 & 15 & 100 \\
8 & 9 & 7 & 77,77 \\
9 & 7 & 7 & 100 \\
10 & 12 & 15 & 125 \\
11 & 7 & 7 & 100 \\
12 & 9 & 9 & 100 \\
13 & 13 & 13 & 100 \\
14 & 12 & 12 & 100 \\
15 & 14 & 14 & 100 \\
\hline
\end{tabular}


Análisis espectral a través de bancos de filtros aplicado al pre-procesamiento para la umbralización de señales de pulso oximetría

Tabla 3. Porcentaje de error. Fuente: autores.

\begin{tabular}{ccc}
\hline Registro & $\begin{array}{c}\text { Promedio de } \\
\text { Porcentaje } \\
\text { de Error }\end{array}$ & $\begin{array}{c}\text { Desviación } \\
\text { Estándar }\end{array}$ \\
\hline 1 & $13,2 \%$ & 3,49 \\
2 & $2,3 \%$ & 1,67 \\
3 & $3 \%$ & 2,3 \\
4 & $1 \%$ & 0,9 \\
5 & $2,5 \%$ & 2,4 \\
6 & $4,5 \%$ & 5,32 \\
7 & $0,22 \%$ & 0,34 \\
8 & $75,34 \%$ & 80 \\
9 & $0,79 \%$ & 0,97 \\
10 & $25,17 \%$ & 25,94 \\
11 & $17,67 \%$ & 6,93 \\
12 & $0,19 \%$ & 0,24 \\
13 & $0,56 \%$ & 0,46 \\
14 & $1,44 \%$ & 0,77 \\
15 & $1,50 \%$ & 1,36 \\
\hline & & \\
\hline & & 0
\end{tabular}

SIN FILTRO

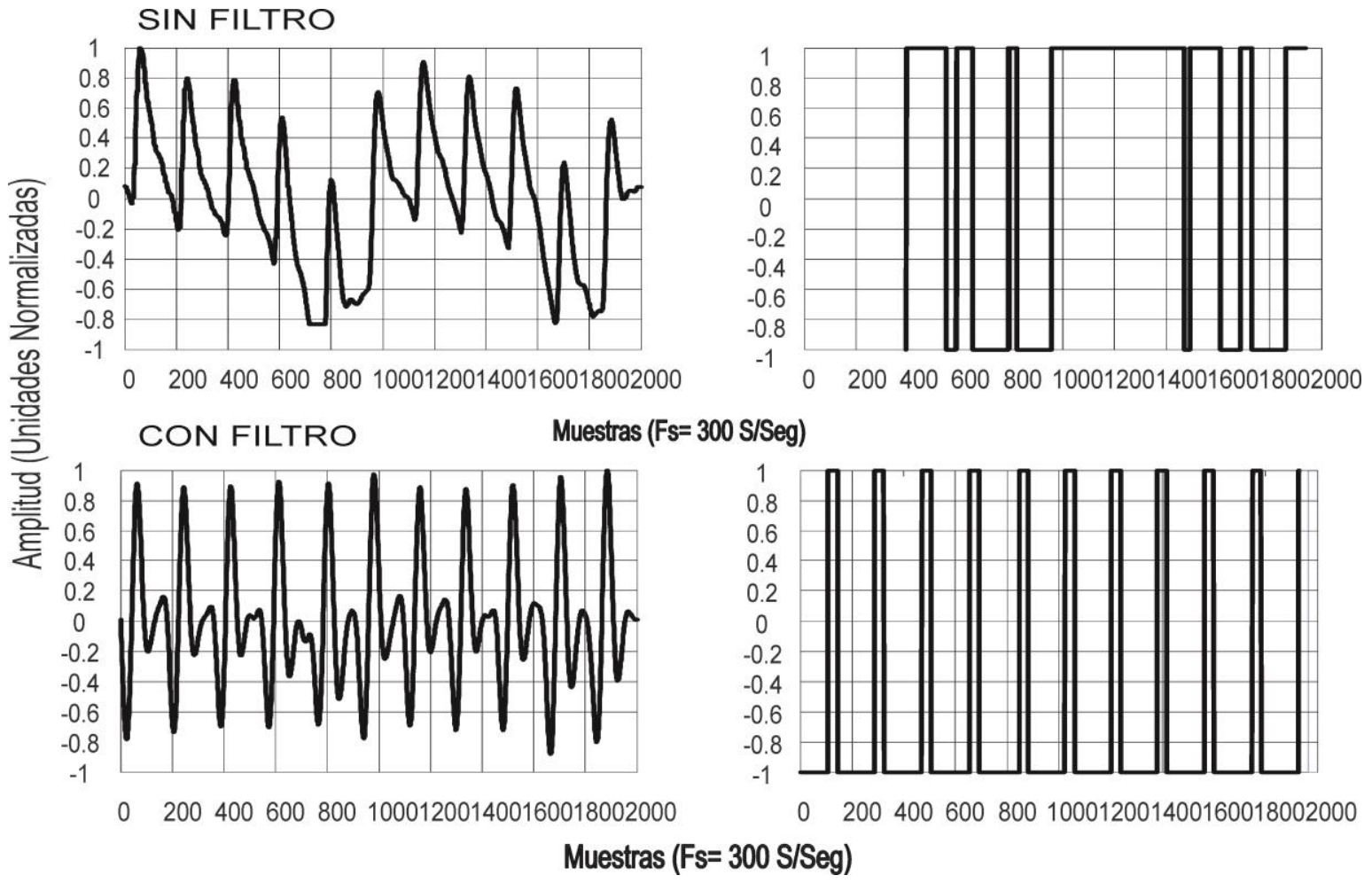

Fig. 13. Comparación del uso del filtro pasa banda en el proceso de umbralización. Fuente: autores.

\begin{tabular}{|c|c|c|c|}
\hline Registro & $\begin{array}{c}\text { Ondas } \\
\text { Presentes }\end{array}$ & $\begin{array}{c}\text { Picos } \\
\text { detectados } \\
\text { (sin filtro) }\end{array}$ & $\begin{array}{c}\text { Picos } \\
\text { detectados } \\
\text { (con filtro) }\end{array}$ \\
\hline 1 & 11 & 6 & 9 \\
\hline 2 & 13 & 0 & 13 \\
\hline 3 & 12 & 0 & 12 \\
\hline 4 & 11 & 12 & 11 \\
\hline 5 & 8 & 8 & 8 \\
\hline 6 & 8 & 7 & 6 \\
\hline 7 & 15 & 0 & 15 \\
\hline 8 & 9 & 10 & 7 \\
\hline 9 & 7 & 7 & 7 \\
\hline 10 & 12 & 3 & 15 \\
\hline 11 & 7 & 0 & 7 \\
\hline 12 & 9 & 9 & 9 \\
\hline 13 & 13 & 0 & 13 \\
\hline 14 & 12 & 0 & 12 \\
\hline 15 & 14 & 14 & 14 \\
\hline
\end{tabular}


Con la finalidad de verificar el valor agregado del banco de filtros se realizó un último experimento, en el cual se pueden comparar los eventos detectados con el proceso de umbralización con la señal SPO2 previamente filtrada y sin el uso del filtro pasa banda. Un ejemplo del resultado obtenido está descrito en la Fig. 13, donde se pueden ver que el uso del filtrado pasa banda contribuye al realce de los picos positivos y permite una correcta detección.

La Tabla 4 contiene una cuantificación de aciertos logrados con el uso del filtro en el proceso de umbralización y sin el uso del filtrado. Los resultados plasmados en la Tabla 4 corroboran la necesidad de un filtrado pasa banda selectiva para garantizar la detección de los picos positivos de la señal SPO2.

Los resultados presentados en este artículo son el producto de experimentos que permiten, en primera instancia, la selección de un rango de frecuencia de filtrado pasa banda orientado al realce de los picos positivos de la señal SPO2. La selección del orden del filtro influye en la calidad de este realce. Los resultados expuestos en las Tablas 2, 3 y 4 permiten verificar la importancia de selección del rango de frecuencias del filtrado pasa banda como método de pre-procesamiento de la señal SPO2 en el proceso de umbralización.

\section{CONCLUSIONES}

El artículo contiene los resultados obtenidos al implementar una metodología de bancos de filtros digitales para facilitar la obtención y detección de picos en señales de oximetría de pulso. Esta metodología permite obtener mejores resultados al realizar mediciones de frecuencia cardiaca sobre la señal de pulsoximetría. Usando algoritmos poco complejos se pueden obtener muy buenos resultados al realizar este tipo de medidas sobre las señales biológicas, este tipo de algoritmos permiten ganar confiabilidad y estabilidad en el sistema, así mismo se pueden asegurar tiempos de respuesta mucho más rápidos y medidas más precisas.

Los resultados presentados ofrecen un valor agregado a trabajos previos relacionados con el proceso de umbralización de señales SPO2. Como se pudo observar en la Tabla 4, la necesidad de un filtro pasa banda incrementa la probabilidad de detección de picos positivos en el proceso de umbralización.

\section{REFERENCIAS}

[1] L. M. Rodriguez Alvarez, "La pulso oximetría en el ámbito prehospitalario," Emergencias, vol. 27, no. 1, pp. 9-10, 2015.

[2] J. P. S. S. M. López Silva, R. Giannetti, M. L. Dotor, D. Golmayo, P. Martín, F. Miguel-Tobal, A. Bilbao, "Fotopletismografía por transmisión con múltiples diodos láser en el infrarrojo cercano durante el ejercicio físico," Óptica pura y Apl., vol. 38, no. 1, pp. 31-39, 2005.

[3] N. Utami, A. W. Setiawan, H. Zakaria, T. R. Mengko, and R. Mengko, "Extracting blood flow parameters from Photoplethysmograph signals: A review," in 2013 3rd International Conference on Instrumentation, Communications, Information Technology and Biomedical Engineering (ICICI-BME), 2013, vol. 1, pp. 403-407.

[4] K. A. Reddy and V. J. Kumar, "Motion Artifact Reduction in Photoplethysmographic Signals using Singular Value Decomposition," in 2007 IEEE Instrumentation \& Measurement Technology Conference IMTC 2007, 2007, vol. 1, pp. 1-4.

[5] J. E. González-Barajas, "Threshold calculation for $\mathrm{R}$ wave detection in complex cardiac," Tecno Lógicas, vol. 
Análisis espectral a través de bancos de filtros aplicado al pre-procesamiento para la umbralización de señales de pulso oximetría

17, no. 32, pp. 47-55, Feb. 2014.

[6] E. C. Lee, Y. Kim, H. Kim, and J. Kim, "Method for restoring PPG signals using ECG correspondences and SVR," Electron. Lett., vol. 49, no. 24, pp. 1518-1520, Nov. 2013.

[7] Z. Zhang, "Heart rate monitoring from wrist-type photoplethysmographic (PPG) signals during intensive physical exercise," in 2014 IEEE Global Conference on Signal and Information Processing (GlobalSIP), 2014, vol. 1, no. 1, pp. 698-702.

[8] R. Laulkar and N. Daimiwal, "Acquisition of PPG signal for diagnosis of parameters related to heart," in 2012 1st International Symposium on Physics and Technology of Sensors (ISPTS-1), 2012, vol. 1, no. 1, pp. 274-277.

[9] A. . C. Banerjee R.; Sinha, "PhotoECG:

Photoplethysmographyto estimate ECG parameters," in Acoustics, Speech and Signal Processing, 2014, vol. 1, no. 1, pp. 4404-4408.

[10] N. Daimiwal, M. Sundhararajan, and R. Shriram, "Respiratory rate, heart rate and continuous measurement of BP using PPG," in 2014 International Conference on Communication and Signal Processing, 2014, vol. 1, no. 1, pp. 999-1002.

[11] K. V. Madhav, M. R. Ram, E. H. Krishna, K. N. Reddy, and K. A. Reddy, "A robust signal processing method for extraction of respiratory activity from artifact corrupted PPG signal," in 2011 IEEE Recent Advances in Intelligent Computational Systems, 2011, vol. 1, no. 1, pp. 451-456.

[12] S. Mohamed Yacin, M. Manivannan, and V. Srinivasa Chakravarthy, "Measurement of gastric oscillations from finger photoplethysmographic signal using autoregressive model," in 2010 International Conference on Communication Control and Computing Technologies, 2010, vol. 1, no. 1, pp. 514-517.

[13] R. W. C. G. R. Wijshoff, A. M. T. M. van Asten, W. H. Peeters, R. Bezemer, G. J. Noordergraaf, M. Mischi, and R. M. Aarts, "Photoplethysmography-Based

Algorithm for Detection of Cardiogenic Output During Cardiopulmonary Resuscitation," IEEE Trans. Biomed. Eng., vol. 62, no. 3, pp. 909-921, Mar. 2015.

[14] N. V. Manaf and T. Kayikcioglu, "Time difference analysis of twowavelength photoplethysmograph signals," in 2014 22nd Signal Processing and Communications Applications Conference (SIU), 2014, vol. 1, no. 1, pp. 2241-2244.

[15] M. R. Ram, K. V. Madhav, E. H. Krishna, K. N. Reddy, and K. A. Reddy, "Use of spectral estimation methods for computation of SpO $<$ inf $>2<$ inf $>$ from artifact reduced PPG signals," in 2011 IEEE Recent Advances in Intelligent Computational Systems, 2011, vol. 1, no. 1, pp. 431-436.

[16] K. V. P. Naraharisetti and M. Bawa, "Comparison of different signal processing methods for reducing artifacts from photoplethysmograph signal," in 2011 IEEE International Conference on Electro/Information Technology, 2011, vol. 1, no. 1, pp. 18.

[17] M. R. Ram, K. V. Madhav, E. H. Krishna, N. R. Komalla, and K. A. Reddy, "A Novel Approach for Motion Artifact Reduction in PPG Signals Based on AS-LMS Adaptive Filter," IEEE Trans. Instrum. Meas., vol. 61, no. 5, pp. 1445-1457, May 2012.

[18] C. Karmakar, A. Khandoker, T. Penzel, C. Schobel, and M. Palaniswami, "Detection of 
Respiratory Arousals Using Photoplethysmography (PPG) Signal in Sleep Apnea Patients," IEEE J. Biomed. Heal. Informatics, vol. 18, no. 3, pp. 1065-1073, May 2014.

[19] L. S. Frida Sandberg, Raquel Bailon, David Hernando, Pablo Laguna, Juan Pablo Martinez, Kristian Solem, "Prediction of intradialytic hypotension using PPG and ECG," Comput. Cardiol. Conf., vol. 1, no. 1, pp. 1227-1230, 2013.

[20] R. P. Arberet S., Lemay M., "Photoplethysmography-based ambulatory heartbeat monitoring embedded into a dedicated bracelet," Comput. Cardiol. Conf., vol. 1, no. 1, pp. 935-938, 2013.

[21] H. S. Shin, C. Lee, and M. Lee, "Adaptive threshold method for the peak detection of photoplethysmographic waveform," Comput. Biol. Med., vol. 39, no. 12, pp. 1145-1152, Dec. 2009.

[22] A. Verma, S. Cabrera, A. Mayorga, and H. Nazeran, "A Robust Algorithm for Derivation of Heart Rate Variability Spectra from ECG and PPG Signals," in 2013 29th Southern Biomedical Engineering Conference, 2013, vol. 1, no. 1, pp. 35-36.
[23] Xiaochuan He, R. A. Goubran, and X. P. Liu, "Secondary Peak Detection of PPG Signal for Continuous Cuffless Arterial Blood Pressure Measurement," IEEE Trans. Instrum. Meas., vol. 63, no. 6, pp. 1431-1439, Jun. 2014.

[24] F. G. Agro D., Canicatti R., Tomasino A., "PPG Embedded System for Blood Pressure Monitoring," in Annual ConferenceFrom Research to Industry: The Need for a More Effective Technology Transfer (AEIT), 2014, vol. 1, no. 1, pp. 1-6.

[25] "CAPNOBASE." [Online]. Available: http://www.capnobase.org/. [Accessed: 26-Jul-2015].

[26] A. Garde, W. Karlen, J. M. Ansermino, and G. A. Dumont, "Estimating Respiratory and Heart Rates from the Correntropy Spectral Density of the Photoplethysmogram," PLoS One, vol. 9, no. 1, p. e86427, Jan. 2014.

[27] S. R. Oppenheim A., Tratamiento de Señales en Tiempo Discreto, 3rd ed. Pearson Education, 2012. 